\title{
Monitoring Walking and Cycling of Middle-Aged to Older Community Dwellers Using Wireless Wearable Accelerometers*
}

\author{
Yuting Zhang ${ }^{1}$, Karel G. M. Beenakker ${ }^{2}$, Pankil M. Butala ${ }^{1}$, Cheng-Chieh Lin ${ }^{3}$, \\ Thomas D.C. Little ${ }^{1}$, Senior Member, IEEE, Andrea B. Maier ${ }^{2}$, Marjon Stijntjes ${ }^{2}$, \\ Richard Vartanian ${ }^{3}$, and Robert C. Wagenaar ${ }^{3}$ \\ ${ }^{1}$ Department of Electrical and Computer Engineering \\ ${ }^{3}$ Department of Physical Therapy and Athletic Training \\ Boston University, Boston, MA 02215, USA \\ $\{$ ytzhang, pbutala, tdcl\}@bu.edu \\ ${ }^{2}$ Department of Gerontology and Geriatrics \\ Leiden University Medical Center, Leiden, 2333 ZA, Netherlands
}

MCL Technical Report No. 08-01-2012

\begin{abstract}
Changes in gait parameters have been shown to be an important indicator of several age-related cognitive and physical declines of older adults. In this paper we propose a method to monitor and analyze walking and cycling activities based on a triaxial accelerometer worn on one ankle. We use an algorithm that can (1) distinguish between static and dynamic functional activities, (2) detect walking and cycling events, (3) identify gait parameters, including step frequency, number of steps, number of walking periods, and total walking duration per day, and (4) evaluate cycling parameters, including cycling frequency, number of cycling periods, and total cycling duration. Our algorithm is evaluated against the triaxial accelerometer data obtained from a group of 297 middle-aged to older adults wearing an activity monitor on the right ankle for approximately one week while performing unconstrained daily activities in the home and community setting. The correlation coefficients between each of detected gait and cycling parameters on two weekdays are all statistically significant, ranging from 0.668 to 0.873 . These results demonstrate good test-retest reliability of our method in monitoring walking and cycling activities and analyzing gait and cycling parameters. This algorithm is efficient and causal in time and thus implementable for real-time monitoring and feedback.
\end{abstract}

In Proc. $34^{\text {th }}$ Annual Intl. Conf. IEEE Engineering in Medicine and Biology Society, August 2012. This material is based upon work support by the Netherlands Consortium for Healthy Aging in Leiden University Medical Center, Coulter Foundation Translational Partners in Biomedical Engineering, and NIH/NIA, Claude Pepper Older Americans Independence Center. 


\section{Introduction}

Healthcare systems are facing many new challenges as the world's population ages. In the United States, the older population, aged 65 and over, numbered 39.6 million in 2009, and is expected to be 72.1 million by $2030 ; 80 \%$ of which have one or more chronic conditions [1]. The national cost for elderly healthcare has been estimated over $\$ 836$ billion [2]. Gait changes in older adults have been shown to be an important indicator of several age-related cognitive and physical declines [3]. Gait characteristics have also been shown associated with health status [3], falls [4], frailty [5], and survival [6]. Older adults at risk display reduced stride length and walking speed, decreased walking endurance and increased gait variability [7]. The long-term monitoring of walking activities in daily life of older adults in the home and community setting has become increasingly important in predicting clinical outcomes and life expectancy that incorporates health and function, and promoting the Quality of Life (QoL) of individual patients. The objectivity and comprehensiveness of activity monitoring is expected to be greatly improved by the use of small, wearable wireless systems that automatically identify and evaluate functional activities such as walking events and gait parameters.

This paper focuses on the development of an efficient algorithm that can (1) classify static and dynamic functional activities, (2) detect walking and cycling events, (3) identify gait parameters, including step frequency, number of steps, number of walking periods, and total walking duration per day, and (4) evaluate cycling parameters, including cycling frequency, number of cycling periods, and total cycling duration, based on the signals from a triaxial accelerometer positioned on each participant's right ankle. This algorithm has been applied to the week-long accelerometer data from unconstrained daily activities of a group of 297 middle-aged to older community dwellers in the home and community setting. The remainder of the paper is organized as follows: the experimental design is explained in Section II; the method to process acceleration data in order to detect walking and cycling events and evaluate gait and cycling parameters is explained in Section III; the results are presented in Section IV; Section V concludes the paper.

\section{Experimental Design}

A prospective longitudinal cohort study investigating predictive factors of longevity and disease in old age was conducted by the Department of Gerontology and Geriatrics, Leiden University Medical Center, the Netherlands. Within the Leiden Longevity Study* families in which at least two long-lived siblings were alive were recruited, fulfilling the age-criterion of 89 years for males and 90 years for females [8]. Furthermore, the children from the long-living subjects, and the partners thereof were included. Written informed consents were obtained from each subject. A group of 297 middle aged to older adults (147 male, 150 females, 45-84 years old, mean age 65.7 years) wore a Gravity Estimator of Normal Everyday Activity (GENEA) just above the lateral malleolus of the right ankle. Subjects were instructed to wear this monitor for a week, consecutive seven days, while they performed unconstrained daily activities in the home and community setting. The GENEA is developed by Unilever Discover (Colworth, United Kindom), manufactured and distributed by ActivInsights Limited (Kimbolton, Cambridgeshire, United Kingdom). It is comprised of a small (36 x $30 \times 12 \mathrm{~mm})$, light weight (16 grams) watch-shaped body-worn triaxial digital accelerometer (dynamic range of \pm 6 gn) with the sampling frequency at $10,20,40$ or $80 \mathrm{~Hz}$ and has the memory capability to save 0.5 Gbyte of raw data. The built-in rechargeable lithium battery can support at least 8 days of reliable data logging at $80 \mathrm{~Hz}$. The week-long raw data were uploaded afterwards and converted into time series matrices in Matlab (MathWorks, Natick, MA, USA) for off-line analysis. 


\section{Methods}

The triaxial accelerometer measures accelerations in the frontal, sagittal and vertical directions from human movements and external perturbations against the Earth's gravity (gn). Fig. 1 illustrates the steps in the algorithm to identify walking and cycling events and parameters from the accelerometer data.

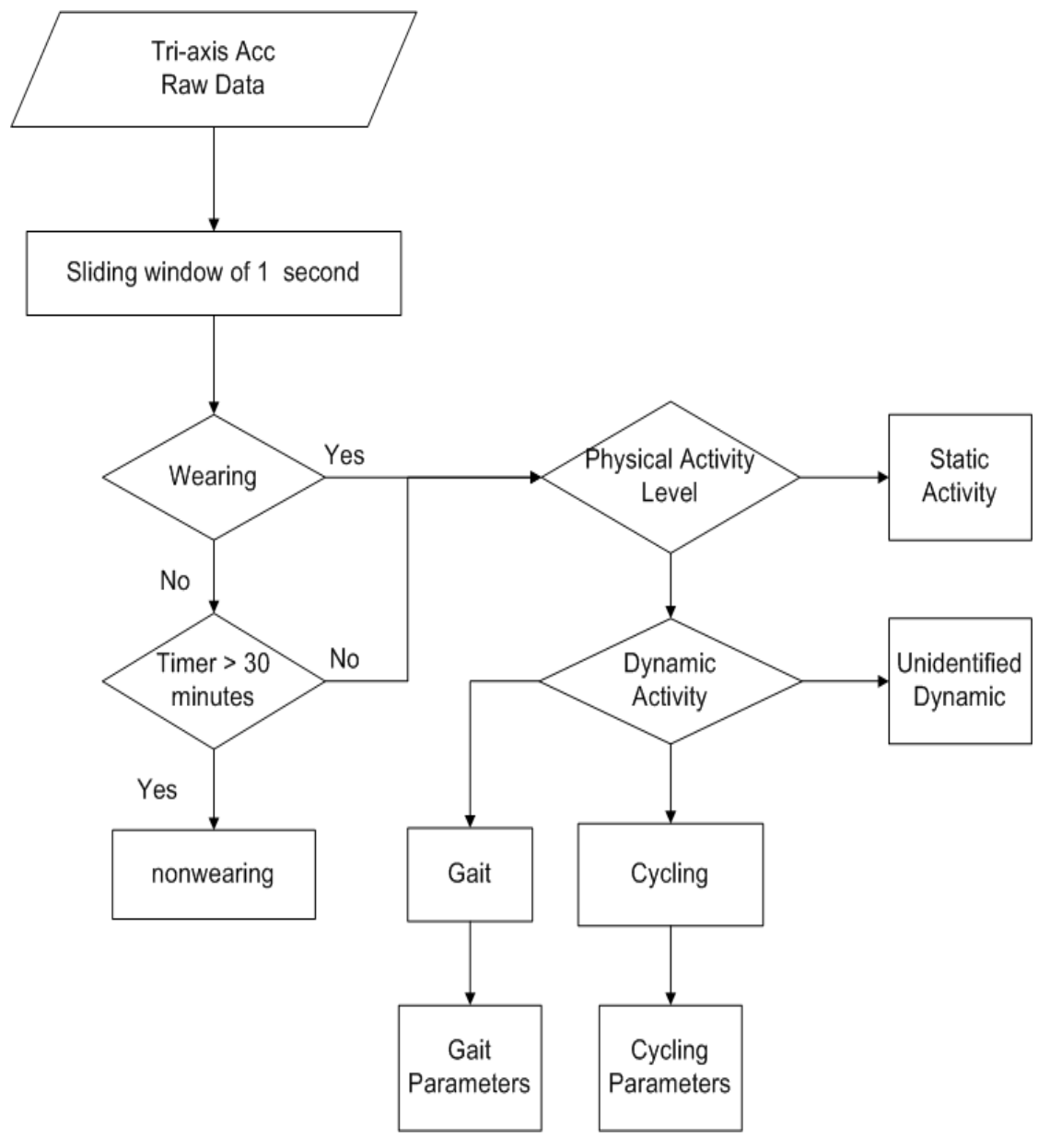

Figure 1. Activity Classification Flowchart

\section{A. Preprocessing}

The raw digital acceleration signals are calibrated to the Earth's gravity. A second-order forward-backward digital low-pass Butterworth filter is applied to the calibrated signals to filter out noises beyond the frequency range of acceleration from human movements.

\section{B. Validation of Wearing}

The validation that the subject is wearing the accelerometer is a prerequisite to monitor and analyze any activity of daily life, such as walking and cycling. We designed and applied a criterion with the following elements to establish wearing time.

(1) Within a time window of $1 \mathrm{~s}$, the square sum of the three axes remains in the range from 0.9 to $1.1 \mathrm{gn} ;$ 
(2) The standard deviation of each axis signal in the same second is less than $0.003 \mathrm{gn}$.

If (1) and (2) are both satisfied in a continuous interval of 30 minutes, the sensor is deemed to be non-wearing during the interval. Otherwise, the accelerometer is regarded as being worn by the participant and functioning properly.

\section{Classification of Static and Dynamic Activities}

Once the accelerometer is validated as being worn by the subject, we classify each $1 \mathrm{~s}$ interval into two coarse-grained categories: (1) static activities, such as standing, sitting, and lying down, or (2) dynamic activities, such as walking, cycling and transitions between activities. Static activities qualitatively show a flatter acceleration time series for all three axes, while dynamic activities yield large differences between consecutive samples. The topmost graph in Fig. 2 shows the accelerometer data in the frontal (X) axis, sagittal (Y) axis and vertical $(\mathrm{Z})$ axis when a participant transits from sitting to standing at approximately $4 \mathrm{~s}$ and begins to walk at $15 \mathrm{~s}$. To capture the differences of variability in time series, a Signal Magnitude Vector (SMV) of each sample point is calculated using eq.(1), which is the square root of the squared sum of the difference of $\mathrm{x}-, \mathrm{y}-$ and $\mathrm{z}$ - values between the current ith sample and its previous (i-1)th sample.

$$
\text { SMVi=2(xi-xi-1)2+(yi-yi-1)2+(zi-zi-1)2 }
$$

The Signal Magnitude Area (SMA) is obtained by summing SMV in each second as eq.(2), where Fs is the sampling frequency of the accelerometer.

$$
\mathrm{SMA}=\mathrm{j}=1 \mathrm{FsSMVj}
$$

The functional activity classified in each second by the threshold test is shown in Fig. 2 .
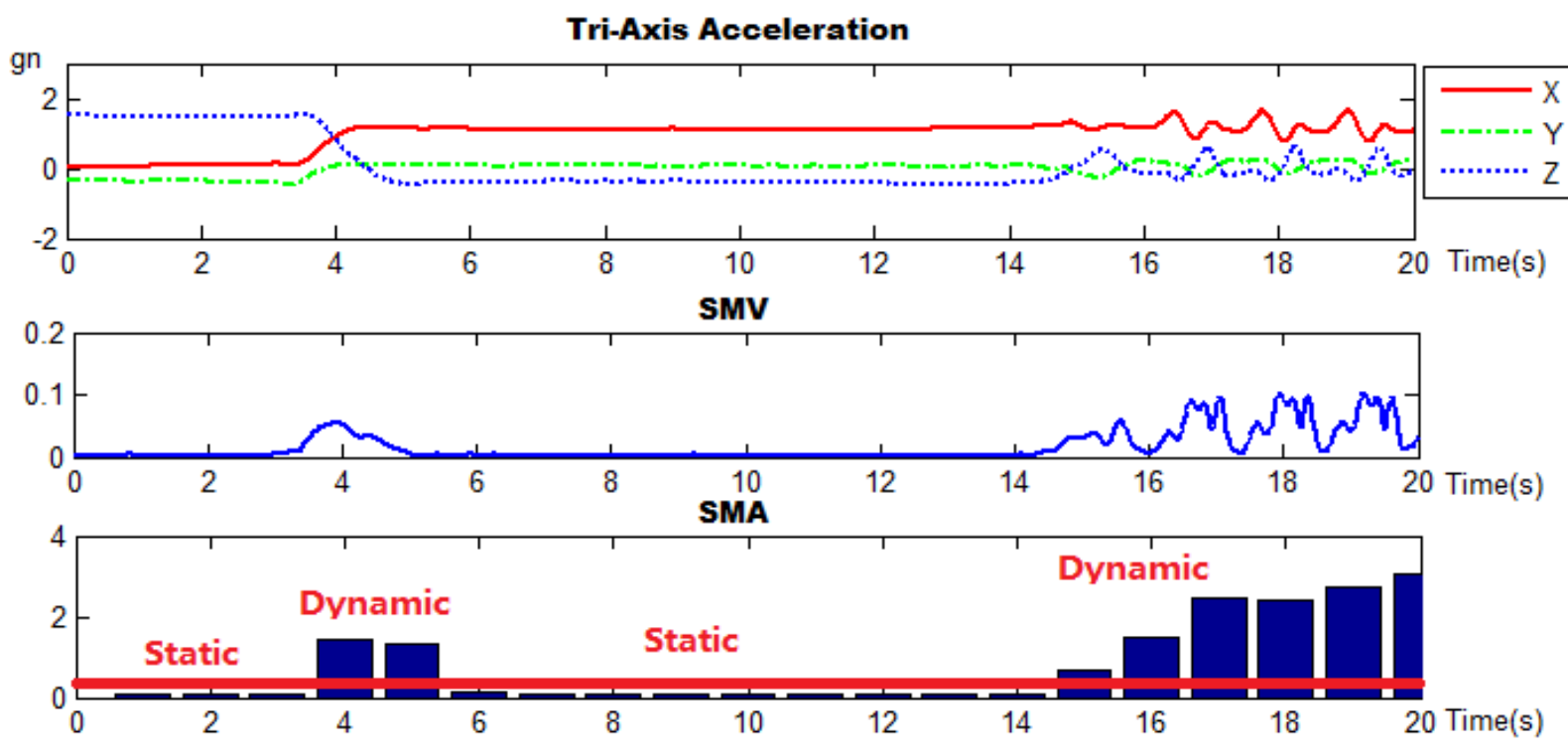

Figure 2. Classification of static and dynamic activities 


\section{Detection of Walking}

Human walking is defined as bipedal, biphasic forward propulsion of centre of mass of human body, in which there are alternate sinuous movements of different segments of the body [9]. Accelerometers can measure accelerations from these movements; particularly the vertical axis is most sensitive to the changes of the center of mass. During walking, a stride cycle begins and ends with the heel strike from the same foot, which is defined as when the heel contacts the ground [9]. A step cycle begins with the heel strike from one foot and ends with that from the other.

Once a consecutive set of five 1s periods is classified as dynamic activities, spectrum analysis is applied to the data of this interval. The Power Spectrum Density (PSD) is estimated by the Fast Fourier Transformation (FFT), using the Welch method with a Hann window [10]. The PSD of the vertical acceleration has significant components at the primary gait frequency (GF) and the secondary GF. The primary GF represents the stride frequency of walking, and the secondary GF represents the step frequency. The acceleration signals of a detected walking event are shown in Fig. 3 with its PSD.

The stride frequency and step frequency of gait events are captured from this PSD. At the end of each walking period, gait parameters including walking duration, the number of steps and strides, and the number of step and strides per minute (cadence) are calculated.
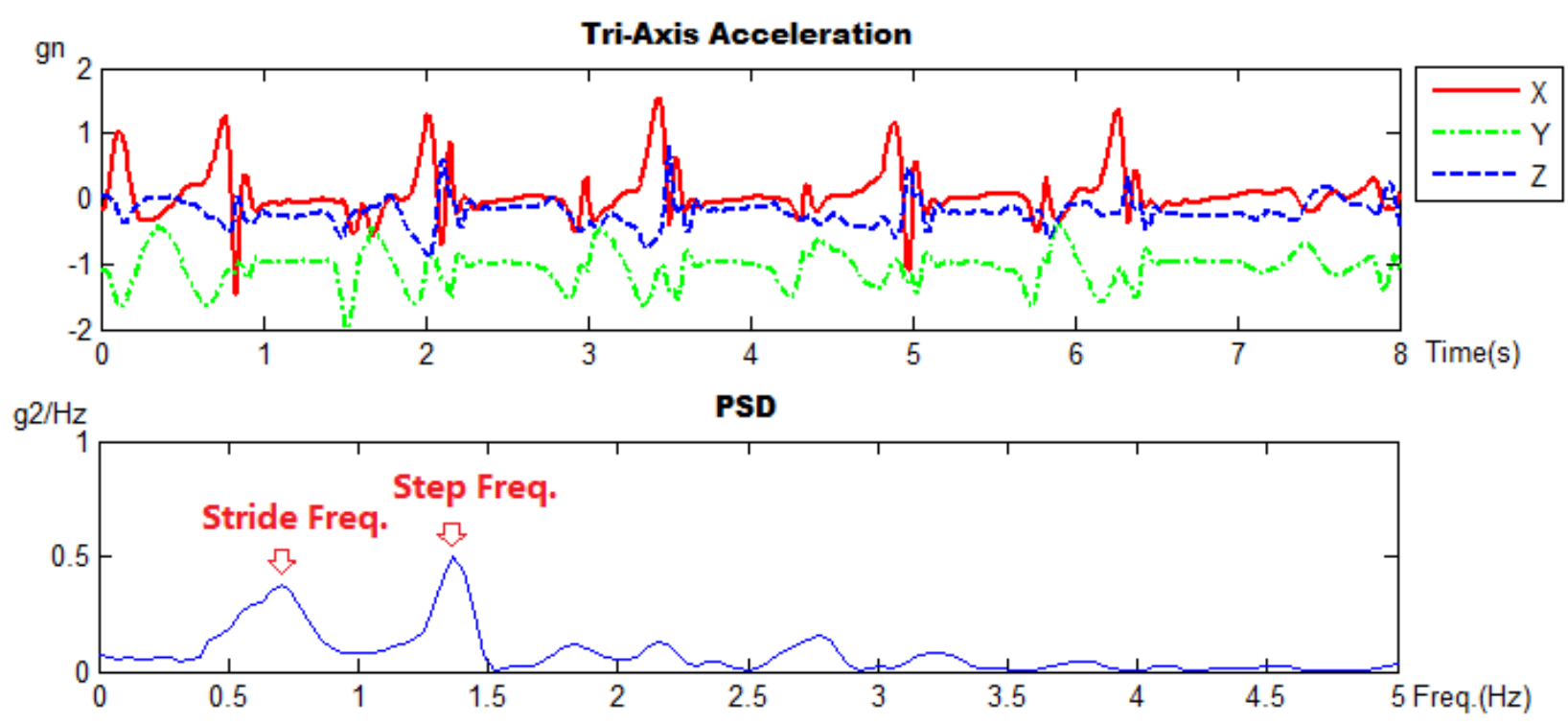

Figure 3. Acceleration of gait and PSD

\section{E. Detection of Cycling}

The cycling activity is another type of common periodic daily activity, which must be distinguished from walking when analyzing parameters of different dynamic activities. The absence of heel-strike/toe-off events and swing/stance phases differentiates the cycling accelerations from walking accelerations in both time series and the PSD. Fig. 4 demonstrates the accelerations in the three axes and the PSD for the vertical axis of a cycling event. The repeatedly constant pedaling leads to the only significant component in the frequency spectrum, evaluated as cycling frequency. 
If the lasting duration of dynamic activity is less than $5 \mathrm{~s}$ or not detected as either walking or cycling event, the time interval is labeled as unidentified dynamic.

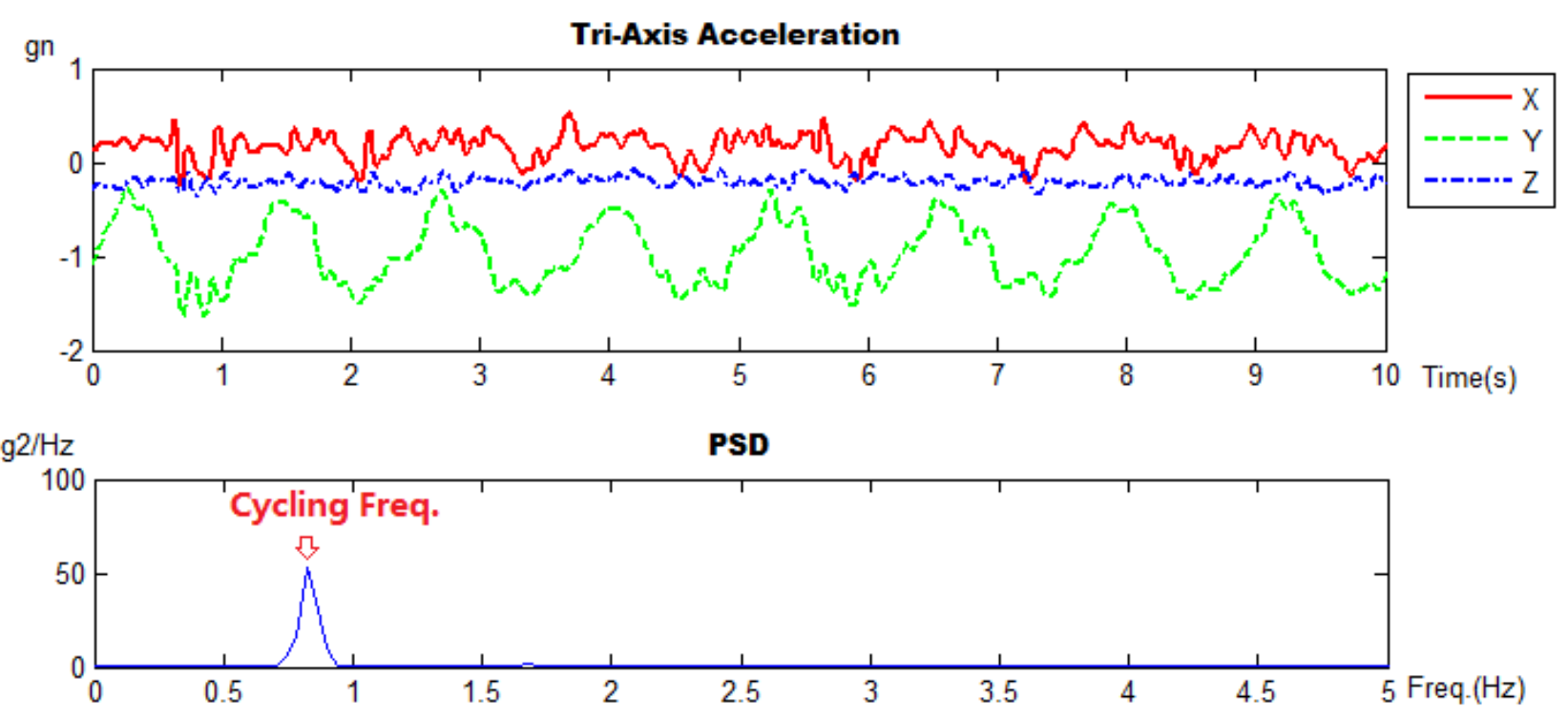

Figure 4. Acceleration of cycling and PSD

\section{Results}

Reliable measurements would detect the consistency in walking and cycling events and parameters between two weekdays, due to the fact that functional activities of an individual remain relatively constant over time. A week of collected data was partitioned into daily sets using the interval of 0:00AM to 11:59PM. We applied our activity classification algorithm to each daily set. For each day, our method identified the following variables with starting and ending time stamp:

(1) Non-wearing time,

(2) Daily walking event and gait parameters of

- w mean step frequency

- w total steps number

- w number of gait periods

- w total gait duration

(3) Cycling events on a subset of 76 subjects and cycling parameters of

- w mean cycling frequency

Based on the measurement starting date and time in the subject log files, comparisons were made between each parameter on two weekdays, using IBM SPSS Statistics 19.0 (IBM, NY, USA). The intraclass correlation (ICC) coefficients were estimated for correlations using a two-way mixed model for absolute agreement. The Wilcoxon signed-rank test was used to evaluate differences between the two weekdays at a 0.05 level of significance. The correlation coefficients in Table I were all statistically significant and ranged from 0.668 for total walking duration to 0.873 for mean step frequency. The test- 
retest results present the failure to reject the null hypothesis that any parameter between two weekdays is consistent by the significance level of 0.05 . Fig. 5 shows scatter plots of each gait parameter on two weekdays, where the horizontal axis stands for the gait parameter on the weekday 1 and the vertical axis for that on the weekday 2. Linear regressions are derived for each scatter plot using Cronbach's Alpha in Table I.

TABLE I. TABLE I. TWO WEEKDAY CORRELATION COEFFICIENTS

\begin{tabular}{|c|c|c|c|c|}
\hline Variable & N & ICC & P value & Cronbach's Alpha \\
\hline Mean Step Frequency & 297 & 0.873 & $<0.001$ & 0.874 \\
\hline Total Steps Number & 297 & 0.681 & $<0.001$ & 0.681 \\
\hline Gait Periods & 297 & 0.733 & $<0.001$ & 0.733 \\
\hline Total Gait Duration & 297 & 0.668 & $<0.001$ & 0.667 \\
\hline Mean Cycling Frequency & 76 & 0.763 & $<0.001$ & 0.761 \\
\hline
\end{tabular}

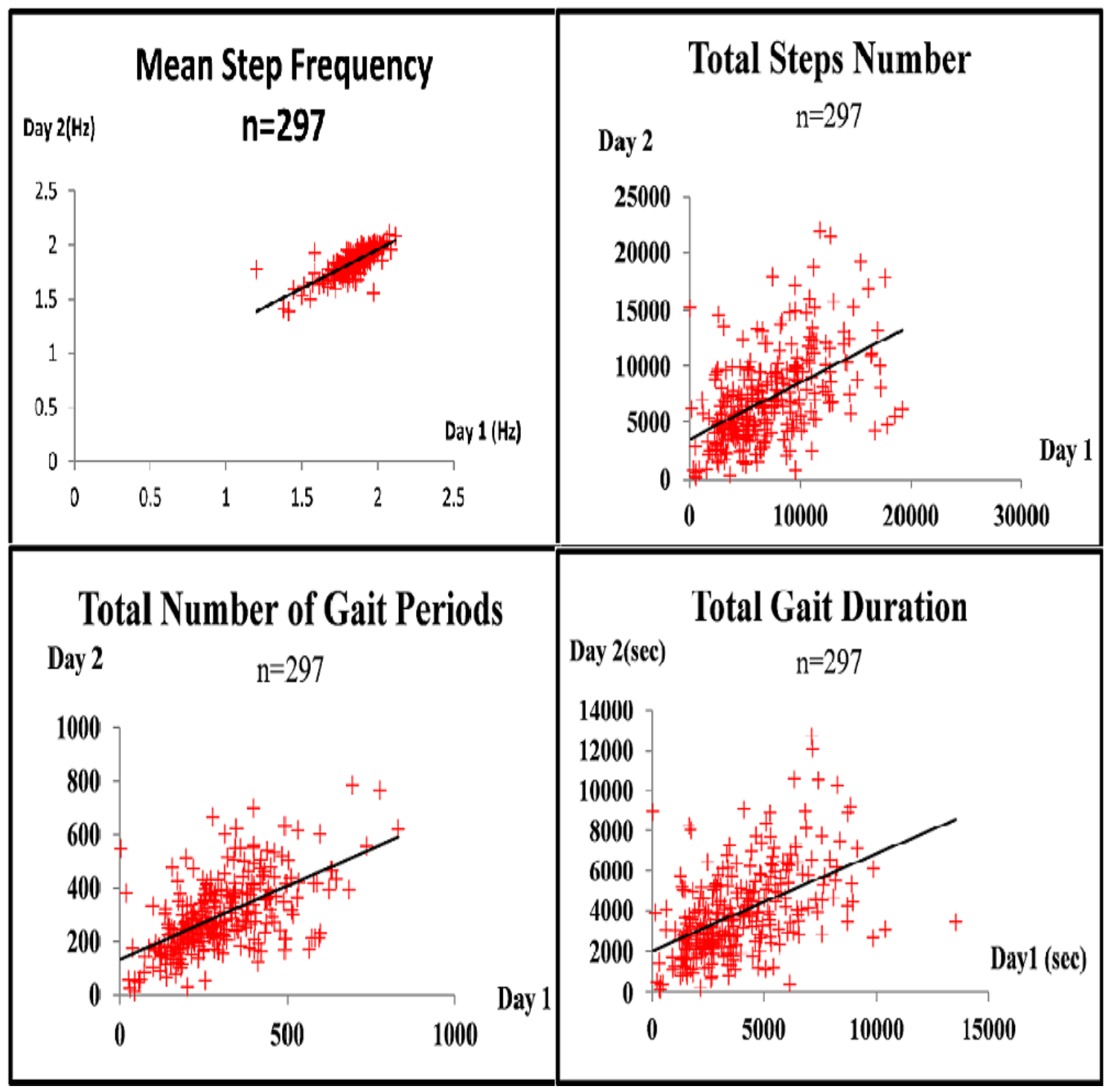

Figure 5. Scatter plots of gait parameters on two weekdays 


\section{Discussion}

The high correlation coefficients demonstrate good test-retest reliability and consistency in the detection of walking and cycling activities, and analysis of gait and cycling parameters, when our method is applied to the ankle accelerometer data obtained from the daily unconstrained functional activities of middle-aged to older community dwellers. Additionally, due to the sequential decision steps, this method is robust in verifying wearing versus non-wearing, and classifying static versus dynamic functional activities.

This classification algorithm is efficient and causal in time and thus implementable on a functional activity monitor (FAM) system for real-time continuous monitoring. The FAM system constitutes multiple low-power body-worn kinematic sensors wirelessly connected to a smartphone [11]. Applying the signal processing decision tree in [12], a software application has been developed for an iPhone (Apple Inc, Cupertino, CA, USA) to provide personal calibration, store sensor signals, trigger alerts when movement disorders occur and continuously identify functional activities including postures, transitions, and walking in the real time.

The device enables augmented therapy provided by the rehabilitation practitioners (e.g., physical therapists) as well as the evaluation and management of functional activities. As one application, the FAM system is capable of continuously detecting unconstrained over-ground walking events, monitoring gait parameters, and providing real-time feedback on the endurance of walking. The smartphone detects the time in seconds when a suggested amount (e.g. 10 minutes) of walking exercise starts and provides an audio feedback and a visual message at the end of the desired time interval to indicate the completion of the endurance exercise.

In our on-going research, we plan to expand our library to monitor a large variety of functional activities, including upper extremity movements. We will explore efficient configurations of different numbers of sensors and at various locations on the body. We will also use the FAM system to monitor changes and progression in functional activities, e.g., the responsiveness of exercise treatment programs. Advantages of using activity monitors include low cost, ease of use and wear by participants, nonintrusiveness, and capability to provide real-time feedbacks for movement disorders. It has tremendous potential to be largely used by older adults in the home and community setting in different perspectives of diagnosis, prognosis, and other related studies of neuromuscular and physical function.

\section{References}

[1] "Healthy Aging: helping People to live long and productive lives and enjoy a good quality of life," National Centers for Disease Control and Prevention, Division of Adult and Community Health, May 2011.

[2] M. W. Stanton, "The high concentration of U. S. health care expenditures," P Rockville, MD: Agency for Healthcare Research and Quality, Jun. 2006.

[3] G. A. Van Kan, Y. Rolland, S. Andrieu, J. Bauer, et al., "Gait speed at usual pace as a predictor of adverse outcomes in community-dwelling older people, an International Academy on Nutrition and Aging (IANA) Task Force," J Nutr Health Aging, vol. 13, no. 10, pp. 881-889, Dec. 2009.

[4] J. H. R. Verghese, R. Holtzer, R. B. Lipton, et al., "Quantitative gait markers and incident fall risk in older adults," J Gerontal A Biol Sci Med Sci, vol. 64, no. 8, pp. 896-901, Aug. 2009.

[5] L. P. Fried, C. M. Lipton, J. Walston, et al., "Frailty in older adults: evidence for a phenotype," $J$ Gerontol A Biol Sci Med Sci, vol. 56, no. 3, pp. 146-156, Mar. 2001. 
[6] S. Studenski, S. Perera, K. Patel, et al., "Gait speed and survival in older adults," JAMA, vol. 305, no. 1, pp. 50-58, Jan, 2011.

[7] J. S. Brach, r. Berthold, R. Craik, et al., "Gait variability in community-dwelling older adults," $J$ American Geriatrics Society, vol. 29, no. 12, pp. 1646-1550, Dec. 2001.

[8] M. Schoenmaker, A.J. de Craen, P.H. de Meijer, et al., "Evidence of genetic enrichment for exceptional survival using a family approach: The Leiden longevity study," Eur. J. Hum. Genet, vol. 14, pp. 79-84, 2006.

[9] J. Perry, Gait analysis: normal and pathological function. Thorofare, NJ: Slack, 1992.

[10] R. C. Wagenaar, and R. E. A van Emmerik, "Resonant frequencies of arms and legs identify different walking patterns," J. Biomechanics, vol. 33, pp. $853-861,2000$.

[11] P. M. Butala, Y. Zhang, R. C. Wagenaar, and T. D. C. Little, "Wireless system for monitoring and real-time classification of functional activity," in Proc. 2nd Workshop on NetHealth 2012, IEEE Comsnets 2012, Bangalore, India, Jan. 2012.

[12] Y. Zhang, I. Sapir, S. Markovic, R. C. Wagenaar, and T.D.C. Little, "Continuous functional activity monitoring based on wearable tri-axial accelerometer and gyroscope", in Proc. 5th Int ICST Conf on Pervasive Computing Technologies for Healthcare (PervasiveHealth 2011), Dublin, Ireland, May 2011.

* Study is under approval of Institutional Review Board (IRB) from Leiden University Medical Center Ethics Committee, the Netherlands. 